\title{
A STUDY OF MONITOR FUNCTIONS FOR TWO-DIMENSIONAL ADAPTIVE MESH GENERATION*
}

\author{
WEIMING $\mathrm{CAO}^{\dagger}$, WEIZHANG HUANG ${ }^{\ddagger}$, AND ROBERT D. RUSSELL ${ }^{\S}$
}

\begin{abstract}
In this paper we study the problem of two-dimensional adaptive mesh generation using a variational approach and, specifically, the effect that the monitor function has on the resulting mesh behavior. The basic theoretical tools employed are Green's function for elliptic problems and the eigendecomposition of symmetric positive definite matrices. Based upon this study, a general strategy is suggested for how to choose the monitor function, and numerical results are presented for illustrative purposes. The three-dimensional case is also briefly discussed. It is noted that the strategy used here can be applied to other elliptic mesh generation techniques as well.
\end{abstract}

Key words. variational mesh generation, mesh adaptation, monitor function, weight function, Green's function

AMS subject classifications. 65M50,65M20, 35K05

PII. S1064827597327656

1. Introduction. One of the major tasks in solving partial differential equations (PDEs) is the adaptive generation of the mesh, or grid. In two (or higher) spatial dimensions, this mesh generation and adaptation is commonly done using the variational approach, specifically, by minimizing a functional of the coordinate mapping $\xi=\xi(x, y), \eta=\eta(x, y)$ between the physical domain $\Omega_{p}$ with variables $x, y$ and the computational domain $\Omega_{c}$ with variables $\xi, \eta$. The functional is chosen so that the minimum is suitably influenced by the desired properties of the solution of the PDE itself. In most applications, this involves balancing several critical properties; e.g., mesh concentration in areas needing high resolution of the physical solution, mesh alignment to some prescribed vector fields, and preservation of the smoothness and the orthogonality of the mesh lines; e.g., see [3, 4, 20]. Obviously, different meshes are generated depending upon how the functional is formulated and how the minimization problem is solved. Due to the complexity of the mesh generation process, and also the fact that the shapes of the physical and computational domains themselves can have a strong effect on the behavior of the coordinate mapping, it is usually very hard to predict the overall resulting mesh behavior from the functional itself. This has led to a situation where a preponderance of mesh generation approaches have been developed in the past, e.g., see $[3,4,8,14,19,20,21]$, yet the understanding of these approaches is relatively limited.

The functionals used in existing variational approaches for mesh generation and adaptation can usually be expressed in the form $[3,11,14,19,21]$

${ }^{*}$ Received by the editors September 19, 1997; accepted for publication (in revised form) June 2, 1998; published electronically May 26, 1999. This research was supported in part by NSERC (Canada) grant OGP-0008781 and NSF (USA) grant DMS-9626107. The computations were done in part on the machines of the Scientific Computing and Visualization Laboratory (supported by NSF (USA) grant DMS-9628626) at the Department of Mathematics, the University of Kansas.

http://www.siam.org/journals/sisc/20-6/32765.html

${ }^{\dagger}$ Department of Mathematics and Statistics, Simon Fraser University, Burnaby, BC V5A 1S6, Canada (wcao@cs.sfu.ca).

${ }^{\ddagger}$ Department of Mathematics, the University of Kansas, Lawrence, KS 66045 (huang@ math.ukans.edu).

$\S$ Department of Mathematics and Statistics, Simon Fraser University, Burnaby, BC V5A 1S6, Canada (rdr@cs.sfu.ca). 


$$
I[\xi, \eta]=\frac{1}{2} \int_{\Omega_{p}} d x d y\left(\nabla \xi^{T} G_{1}^{-1} \nabla \xi+\nabla \eta^{T} G_{2}^{-1} \nabla \eta\right),
$$

where $G_{1}=G_{1}(x, y)$ and $G_{2}=G_{2}(x, y)$ are symmetric positive definite matrices, referred to as the monitor functions, and $\nabla=\left(\frac{\partial}{\partial x}, \frac{\partial}{\partial y}\right)^{T}$. For simplicity, we consider in this paper the simpler yet still fairly general class of functionals of the form

$$
I[\xi, \eta]=\frac{1}{2} \int_{\Omega_{p}} d x d y\left(\nabla \xi^{T} G^{-1} \nabla \xi+\nabla \eta^{T} G^{-1} \nabla \eta\right),
$$

where $G=G(x, y)$ is a symmetric positive definite matrix. As will be clear later, our strategy used for understanding (1.2) can be straightforwardly applied to (1.1). With (1.2), the coordinate transformation and the mesh are determined from the Euler-Lagrange equation

$$
\begin{aligned}
& \nabla \cdot\left(G^{-1} \nabla \xi\right)=0 \\
& \nabla \cdot\left(G^{-1} \nabla \eta\right)=0
\end{aligned}
$$

or its transformed form (via interchanging the roles of the dependent and independent variables)

$$
\begin{aligned}
& {\left[\frac{\partial}{\partial \xi}\left(\frac{1}{g J} \vec{x}_{\eta}^{T} G \vec{x}_{\eta}\right)-\frac{\partial}{\partial \eta}\left(\frac{1}{g J} \vec{x}_{\xi}^{T} G \vec{x}_{\eta}\right)\right] \vec{x}_{\xi}} \\
& +\left[-\frac{\partial}{\partial \xi}\left(\frac{1}{g J} \vec{x}_{\eta}^{T} G \vec{x}_{\xi}\right)+\frac{\partial}{\partial \eta}\left(\frac{1}{g J} \vec{x}_{\xi}^{T} G \vec{x}_{\xi}\right)\right] \vec{x}_{\eta}=0,
\end{aligned}
$$

supplemented with suitable boundary conditions. Here, $\vec{x}=(x, y)^{T}$ is the physical coordinate, $g=\operatorname{det}(G)$, and $J=x_{\xi} y_{\eta}-x_{\eta} y_{\xi}$ is the Jacobian of the coordinate transformation. Functional (1.2) includes as particular examples Winslow's well-known method and the method based upon harmonic maps. The functional for Winslow's method is

$$
I[\xi, \eta]=\frac{1}{2} \int_{\Omega_{p}} d x d y \frac{1}{w(x, y)}\left(|\nabla \xi|^{2}+|\nabla \eta|^{2}\right),
$$

where $w(x, y)$ is a weight function depending on the physical solution to be adapted, and this corresponds to (1.2) with the monitor function

$$
G=w(x, y) I .
$$

Brackbill and Saltzman [4] generalize Winslow's method to produce satisfactory mesh concentration while maintaining relatively good smoothness and orthogonality. Theirs has become one of the most popular methods used for mesh generation and adaptation.

The method based upon harmonic maps uses the functional

$$
I[\xi, \eta]=\frac{1}{2} \int_{\Omega_{p}} d x d y \frac{1}{\sqrt{m}}\left(\nabla \xi^{T} M^{-1} \nabla \xi+\nabla \eta^{T} M^{-1} \nabla \eta\right),
$$

where $m=\operatorname{det}(M(x, y))$ and $M(x, y)$ is a symmetric positive definite matrix. It can be written in the general form (1.2) with

$$
G=\frac{M}{\sqrt{m}} .
$$


A nice feature of this type of method is that for two-dimensional problems, existence, uniqueness, and nonsingularity for the continuous map can be guaranteed from the theory of harmonic maps (e.g., see $[6,9]$, and see $[15,16]$ for discussion on singularity of three-dimensional harmonic maps), and such theoretical guarantees are rare in the field of mesh generation.

The success of a mesh adaptation strategy with functional (1.2) (and all other methods using a variational approach or an elliptic equation system) hinges on choosing an appropriate monitor function $G$, so understanding how the monitor function influences the resulting mesh properties is clearly crucial. There has been only limited study of this aspect of the adaptive mesh generation problem, e.g., see $[1,2,7,14,18]$, although practical experience has undoubtedly led to insight into a suitable choice for the monitor function in some cases such as in $[3,4,20]$. Our objective in this paper is to more systematically analyze the adaptivity effects for the monitor function $G$ in $(1.2)$.

We find that, in general, the eigenvectors of $G$ determine the directions of the mesh concentration, while the eigenvalues determine the strength of mesh compression or expansion. This insight motivates how $G$ can be chosen in practice. For instance, it can be fortuitous for problems in fluid dynamics to choose an eigenvector to be the streamline direction or the characteristic direction, and for general problems with steep wave fronts to choose an eigenvector in the gradient direction. The eigenvalues are in turn chosen to vary the strength of the mesh concentration.

An outline of the paper is as follows. In section 2, the Dirichlet problem for a general elliptic PDE is considered. The influence of the source term on the solution behavior is discussed both analytically and geometrically using Green's function. Then the eigendecomposition of the monitor function is used in section 3 to investigate the effect of the monitor function on the behavior of the mesh corresponding to the functional form (1.2). The above-mentioned general guide for the choice of the monitor function is also developed in this section. After numerical examples for common choices of the monitor function are presented in section 4 , the case of adaptive mesh generation for a disk is studied in section 5 in order to illustrate some of the complicated two-dimensional effects for mesh adaptation along the eigendirections of the monitor functions. A brief discussion of the three-dimensional case is given in section 6 . Finally, section 7 contains the conclusions.

2. Green's function. Our basic tools for providing insight into the mesh behavior are Green's function for elliptic PDEs and the eigendecomposition of symmetric matrices. Using Green's function, we show in this section how the source function for an elliptic PDE influences the behavior of the solution. These results are crucial to the analysis of the influence of monitor functions on the mesh behavior developed in the next section. Detailed mathematical discussion of Green's function and its applications appears in many places, e.g., in $[5,17]$, but here the main purpose is to examine certain geometrical effects.

Consider the differential operator

$$
L \equiv \sum_{i, j=1}^{n} a_{i j}(\vec{x}) \frac{\partial^{2}}{\partial x_{i} \partial x_{j}}+\sum_{i=1}^{n} b_{i}(\vec{x}) \frac{\partial}{\partial x_{i}},
$$

where the coefficients $a_{i j}=a_{j i}$ and $b_{i}$ are continuous functions of $\vec{x}=\left(x_{1}, \ldots, x_{n}\right)^{T}$ in a bounded domain $\Omega$ in $n$-dimensional space. The quadratic form $\sum_{i, j=1}^{n} a_{i j} \xi_{i} \xi_{j}$ is assumed to be positive definite in the parameter $\vec{\xi}=\left(\xi_{1}, \ldots, \xi_{n}\right)^{T}$ for all $\vec{x} \in \Omega$. 
If the boundary $\partial \Omega$ is sufficiently smooth, then Green's function for the differential operator $L$, denoted by $\mathcal{G}=\mathcal{G}(\vec{x}, \vec{y})$, exists in $\Omega$. Furthermore, $\mathcal{G}$ is positive in $\Omega$ and zero on $\partial \Omega$ and has the asymptotic behavior that as $\vec{y} \rightarrow \vec{x}, \mathcal{G}(\vec{x}, \vec{y}) \rightarrow|\vec{x}-\vec{y}|^{2-n}$ for $n>2$ or $\log |\vec{x}-\vec{y}|$ for $n=2$, where $|\vec{x}-\vec{y}|$ is the distance from $\vec{x}$ to $\vec{y}$. For the Dirichlet problem

$$
\begin{cases}L[u]=f & \text { in } \Omega \\ u=h & \text { on } \partial \Omega\end{cases}
$$

the solution can be expressed by

$$
u(\vec{x})=-\int_{\Omega} \mathcal{G}(\vec{x}, \vec{y}) f(\vec{y}) d \vec{y}-\int_{\vec{y} \in \partial \Omega} h(\vec{y}) \frac{\partial \mathcal{G}}{\partial \vec{n}}(\vec{x}, \vec{y}) d S,
$$

where $\frac{\partial \mathcal{G}}{\partial \vec{n}}=\sum_{i, j=1}^{n} a_{i j} \cos \left(\vec{n}, \vec{e}_{i}\right) \frac{\partial}{\partial x_{j}}, \vec{n}$ is the outward normal to the boundary $\partial \Omega$, and $\vec{e}_{i}$ is the unit vector in the $x_{i}$ axis.

To consider the effect the function $f$ has on the behavior of the solution $u$, define $v$ as the solution of the "homogeneous" problem

$$
\begin{cases}L[v]=0 & \text { in } \Omega \\ v=h & \text { on } \partial \Omega\end{cases}
$$

Clearly,

$$
v(\vec{x})=-\int_{\vec{y} \in \partial \Omega} h(\vec{y}) \frac{\partial \mathcal{G}}{\partial \vec{n}}(\vec{x}, \vec{y}) d S,
$$

and it follows from $(2.3),(2.5)$, and the positivity of $\mathcal{G}(\vec{x}, \vec{y})$ that

$$
f \geq 0 \text { in } \Omega \quad \Longrightarrow \quad u \leq v \text { in } \Omega .
$$

This is of course the well-known conclusion of the comparison theorem [5]. For geometric meaning of the inequality $u \leq v$, Fig. 2.1 gives a sketch of $u$ - and $v$-contour lines (surfaces when $n>2$ ) on which $u$ and $v$ are constant. Since $u(\vec{x}) \leq v(\vec{x})$ for all $\vec{x} \in \Omega$, for any constant $c$ the line $u=c$ lies in the region where $v \geq c$. Therefore, the $u$-contour lines are shifted away from the "uniform" reference $v$-contour lines in the direction toward which $u$ increases. Generally, a large positive value of $f$ causes $u$-contour lines to move in the direction of increasing $u$, and a large negative value causes them to move in the direction of decreasing $u$. The larger the magnitude of $|f|$, the greater the shift.

When the function $f(\vec{x})$ changes sign over the domain, the situation becomes more complicated. To see the influence of such a source function, we consider the extreme (point charge) case

$$
f(\vec{x})=\delta\left(\vec{x}-\vec{x}_{1}\right)-\delta\left(\vec{x}-\vec{x}_{2}\right),
$$

where $\vec{x}_{1}$ and $\vec{x}_{2}$ are two distinct points in the domain and $\delta$ is the Dirac delta function. (We note that this function fails to give a solution $u$ in the strong sense, but it can be smoothed and have sufficiently small compact support that the following discussion can be made rigorous.) In this case, the solution to (2.2) is

$$
u(\vec{x})=-\mathcal{G}\left(\vec{x}, \vec{x}_{1}\right)+\mathcal{G}\left(\vec{x}, \vec{x}_{2}\right)+v(\vec{x}) .
$$




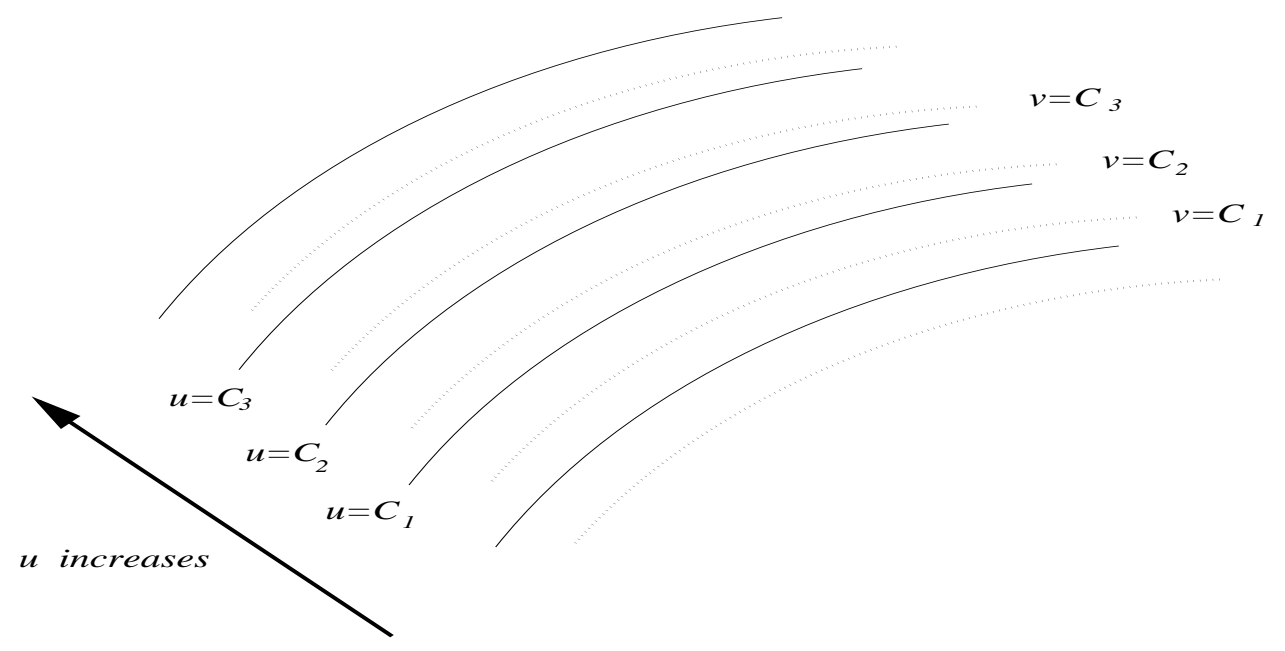

FIG. 2.1. A sketch of $u$ - and $v$-contour lines, where $u<v$ in $\Omega$ and $C_{1}<C_{2}<C_{3}$ are three arbitrary constants.

Since $\mathcal{G}(\vec{x}, \vec{y}) \rightarrow+\infty$ as $\vec{y} \rightarrow \vec{x},(2.8)$ implies that $(u-v)(\vec{x}) \rightarrow-\infty$ as $\vec{x} \rightarrow \vec{x}_{1}$ and $(u-v)(\vec{x}) \rightarrow+\infty$ as $\vec{x} \rightarrow \vec{x}_{2}$. In other words, the influence of the function $f$ on the behavior of $u$ comes predominantly from the value $f\left(\vec{x}_{1}\right)$ near $\vec{x}_{1}$ and from the value $f\left(\vec{x}_{2}\right)$ near $\vec{x}_{2}$. Geometrically, this means that in a neighborhood of $\vec{x}_{1}, f$ causes $u$-contour lines to shift (relative to the $v$-contour lines) in the direction of increasing $u$, and in a neighborhood of $\vec{x}_{2}$ in the direction of decreasing $u$. As a result, the $u$-contour lines in the region between $\vec{x}_{1}$ and $\vec{x}_{2}$ are compressed when $u$ increases in the direction from $\vec{x}_{1}$ to $\vec{x}_{2}$ and are expanded when $u$ decreases. An illustration is given in Fig. 2.2.

This analysis of the point charge source function can be extended to a general function $f$. Because of the singular behavior of Green's function $\mathcal{G}(\vec{x}, \vec{y})$ at $\vec{y}=\vec{x}$, the main contribution to the first integral on the right-hand side of (2.3) comes from the values of $f$ near $\vec{x}$. Thus, $(u-v)$ becomes negative in the region where $f$ is sufficiently large and positive. Geometrically, $u$-contour lines will shift from the reference $v$ contour lines in the increasing $u$ direction in the region. A similar interpretation can of course be made if $(u-v)$ becomes positive in the region where $f$ is sufficiently large and negative. Consequently, $u$-contour lines will be compressed or expanded about the region where $f$ changes sign.

We have thus far assumed that $f$ is a function of $\vec{x}$ only. It is not difficult to see that the analysis in this section holds for the more general case where $f$ depends also upon the unknown function $u$; i.e., $f=f(\vec{x}, u)$. In this case, the sign of $f$ at a point $\vec{x}$ is regarded as the sign of $f(\vec{x}, u(\vec{x}))$, where $u=u(\vec{x})$ is the solution of $(2.2)$.

3. Monitor function in two dimensions. We now use the geometrical interpretations in the previous section to analyze the behavior of the function minimizing the functional (1.2), or the solution of the Euler-Lagrange equation (1.3). Being symmetric and positive definite, the monitor function $G$ and its inverse $G^{-1}$ in (1.2) have the eigendecompositions

$$
\begin{aligned}
G & =\lambda_{1} \vec{v}_{1} \vec{v}_{1}^{T}+\lambda_{2} \vec{v}_{2} \vec{v}_{2}^{T}, \\
G^{-1} & =\lambda_{1}^{-1} \vec{v}_{1} \vec{v}_{1}^{T}+\lambda_{2}^{-1} \vec{v}_{2} \vec{v}_{2}^{T},
\end{aligned}
$$




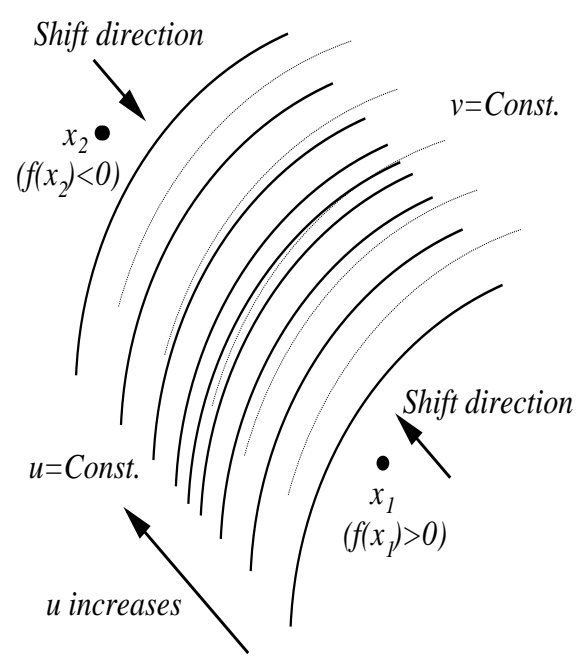

(a) Compression

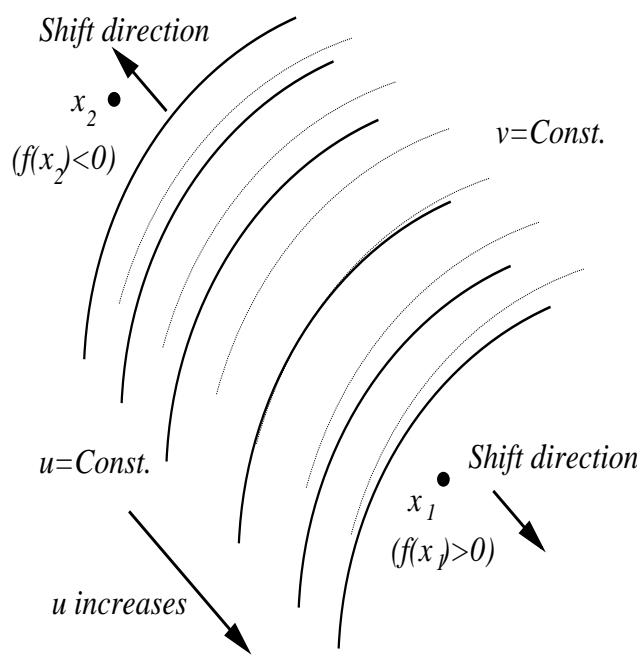

(b) Expansion

FIG. 2.2. Compression and expansion of $u$-contour lines caused by the sources $f\left(\vec{x}_{1}\right)$ and $f\left(\vec{x}_{2}\right)$. ( $u$ - and $v$-contour lines are solid and dotted lines, respectively.)

where $\lambda_{1}$ and $\lambda_{2}$ are the two positive eigenvalues of $G$ and $\vec{v}_{1}$ and $\vec{v}_{2}$ are corresponding normalized orthogonal eigenvectors. Using this decomposition and recalling that the directional derivative in the $\vec{v}$ direction is defined as $\frac{\partial}{\partial \vec{v}}=\vec{v} \cdot \nabla$, we can rewrite the functional (1.2) as

$$
I[\xi, \eta]=\frac{1}{2} \int_{\Omega_{p}} d x d y\left[\frac{1}{\lambda_{1}}\left(\left|\frac{\partial \xi}{\partial \vec{v}_{1}}\right|^{2}+\left|\frac{\partial \eta}{\partial \vec{v}_{1}}\right|^{2}\right)+\frac{1}{\lambda_{2}}\left(\left|\frac{\partial \xi}{\partial \vec{v}_{2}}\right|^{2}+\left|\frac{\partial \eta}{\partial \vec{v}_{2}}\right|^{2}\right)\right]
$$

The Euler-Lagrange equation (1.3) is

$$
\begin{aligned}
& \nabla \cdot\left(\frac{\vec{v}_{1}}{\lambda_{1}} \frac{\partial \xi}{\partial \vec{v}_{1}}\right)+\nabla \cdot\left(\frac{\vec{v}_{2}}{\lambda_{2}} \frac{\partial \xi}{\partial \vec{v}_{2}}\right)=0, \\
& \nabla \cdot\left(\frac{\vec{v}_{1}}{\lambda_{1}} \frac{\partial \eta}{\partial \vec{v}_{1}}\right)+\nabla \cdot\left(\frac{\vec{v}_{2}}{\lambda_{2}} \frac{\partial \eta}{\partial \vec{v}_{2}}\right)=0
\end{aligned}
$$

which after some manipulation becomes

$$
\frac{1}{\lambda_{1}} \frac{\partial^{2} \xi}{\partial \vec{v}_{1}^{2}}+\frac{1}{\lambda_{2}} \frac{\partial^{2} \xi}{\partial \vec{v}_{2}^{2}}=\frac{1}{\lambda_{1}}\left(\frac{1}{\lambda_{1}} \frac{\partial \lambda_{1}}{\partial \vec{v}_{1}}-\nabla \cdot \vec{v}_{1}\right) \frac{\partial \xi}{\partial \vec{v}_{1}}+\frac{1}{\lambda_{2}}\left(\frac{1}{\lambda_{2}} \frac{\partial \lambda_{2}}{\partial \vec{v}_{2}}-\nabla \cdot \vec{v}_{2}\right) \frac{\partial \xi}{\partial \vec{v}_{2}},
$$

$$
\frac{1}{\lambda_{1}} \frac{\partial^{2} \eta}{\partial \vec{v}_{1}^{2}}+\frac{1}{\lambda_{2}} \frac{\partial^{2} \eta}{\partial \vec{v}_{2}^{2}}=\frac{1}{\lambda_{1}}\left(\frac{1}{\lambda_{1}} \frac{\partial \lambda_{1}}{\partial \vec{v}_{1}}-\nabla \cdot \vec{v}_{1}\right) \frac{\partial \eta}{\partial \vec{v}_{1}}+\frac{1}{\lambda_{2}}\left(\frac{1}{\lambda_{2}} \frac{\partial \lambda_{2}}{\partial \vec{v}_{2}}-\nabla \cdot \vec{v}_{2}\right) \frac{\partial \eta}{\partial \vec{v}_{2}} .
$$


Consider now the effect a change in $\lambda_{1}$ has on the behavior of $\xi$ : Rewriting (3.5) in the form (2.2), with

$$
\begin{aligned}
L[\xi] \equiv & \frac{\partial^{2} \xi}{\partial \vec{v}_{1}^{2}}+\left(\frac{\lambda_{1}}{\lambda_{2}}\right) \frac{\partial^{2} \xi}{\partial \vec{v}_{2}^{2}} \\
& +\left(\nabla \cdot \vec{v}_{1}\right) \frac{\partial \xi}{\partial \vec{v}_{1}}+\left(\frac{\lambda_{1}}{\lambda_{2}}\right)\left(\nabla \cdot \vec{v}_{2}\right) \frac{\partial \xi}{\partial \vec{v}_{2}}-\left(\frac{\lambda_{1}}{\lambda_{2}}\right)\left(\frac{1}{\lambda_{2}} \frac{\partial \lambda_{2}}{\partial \vec{v}_{2}}\right) \frac{\partial \xi}{\partial \vec{v}_{2}}, \\
f(x, y) \equiv & \frac{1}{\lambda_{1}} \frac{\partial \lambda_{1}}{\partial \vec{v}_{1}} \frac{\partial \xi}{\partial \vec{v}_{1}}
\end{aligned}
$$

we easily show that the operator $L$ has a form as in (2.1) and is elliptic. Thus, the analysis in the previous section applies. Moreover, since $f$ is proportional to $\frac{1}{\lambda_{1}} \frac{\partial \lambda_{1}}{\partial \vec{v}_{1}}$, a rapid change in $\lambda_{1}$ in the $\vec{v}_{1}$ direction will result in a significant change in $f$ in the same direction. One can then conclude from the analysis in section 2 that if $\lambda_{1}$ changes rapidly in the $\vec{v}_{1}$ direction, $\xi$-coordinate lines can be expected to compress or expand in this direction compared with the reference coordinate lines corresponding to not having the source term $\frac{1}{\lambda_{1}} \frac{\partial \lambda_{1}}{\partial \vec{v}_{1}} \frac{\partial \xi}{\partial \vec{v}_{1}}$. Specifically, one can easily see from the special form of $f$ that compression of $\xi$-coordinate lines occurs in the $\vec{v}_{1}$ direction if $\lambda_{1}$ first increases and then decreases along this direction, while $\xi$-coordinate lines will be expanded if the $\lambda_{1}$ change is the reverse.

We emphasize that this compression and/or expansion of $\xi$-coordinate lines caused by the change in $\lambda_{1}$ is relative motion compared with the reference coordinate lines. To determine the location of the actual $\xi$-coordinate lines, the impact of the other terms in the operator $L$ in (3.7) on the mesh adaptation in the $\vec{v}_{1}$ direction must be taken into account. These include the change in $\vec{v}_{1}$ (the third term), the change in $\vec{v}_{2}$ (the fourth term), the relative change in $\lambda_{2}$ in the $\vec{v}_{2}$ direction (the fifth term), and the ellipticity of the underlying equation (the second term). The latter, the effect of the ellipticity, is well understood - ellipticity tends to space coordinate lines more equally in the absence of boundary curvature. However, the effects from changes in $\vec{v}_{1}, \vec{v}_{2}$, and $\lambda_{2}$ are complicated and difficult to analyze in general. We do not perform a detailed theoretical analysis of these effects here; rather, they are illustrated for several examples in sections 4 and 5. We refer to all of them as two-dimensional effects on the mesh adaptation in the $\vec{v}_{1}$ direction. It is worth pointing out that a decrease of the ratio $\frac{\lambda_{1}}{\lambda_{2}}$ reduces all of the two-dimensional effects on the mesh adaptation in the $\vec{v}_{1}$ direction except that resulting from the change in $\vec{v}_{1}$ (see (3.7)).

A similar analysis holds for the $\xi$-coordinate line adaptation in the $\vec{v}_{2}$ direction and the $\eta$-coordinate lines in the $\vec{v}_{1}$ and $\vec{v}_{2}$ directions. It is useful to note that if, for instance, $\lambda_{1}$ first increases and then decreases rapidly in the $\vec{v}_{1}$ direction, then both the $\xi$-and $\eta$-coordinate lines will be compressed in the same direction and the resulting mesh can become very skewed. The analysis also extends to the case where $G_{2} \neq G_{1}$ in (1.1), in which $\xi$ - and $\eta$-coordinate lines can have different compression and/or expansion directions.

The above shows how the monitor function can be naturally defined directly in terms of suitably chosen $\vec{v}_{1}, \vec{v}_{2}, \lambda_{1}$, and $\lambda_{2}$ using (3.1). Since $\vec{v}_{1}$ and $\vec{v}_{2}$ are orthogonal, the monitor function can be expressed as

$$
G=\lambda_{1} \vec{v} \vec{v}^{T}+\lambda_{2} \vec{v}_{\perp} \vec{v}_{\perp}^{T} .
$$

If one wishes the mesh concentration or expansion to occur mainly along a direction, e.g., the streamline direction in the convection-diffusion problem or the gradient 
direction of the numerical solution, then one may choose this direction as the eigenvector $\vec{v}$, and choose the corresponding eigenvalue $\lambda_{1}$ increasing and then decreasing (or vice versa) in certain regions to give mesh concentration (or expansion) along the $\vec{v}$ direction in the regions. Similarly, $\lambda_{2}$ can be chosen to give mesh concentration or expansion along the $\vec{v}_{\perp}$ direction. Since the resulting mesh is affected by both the changes of $\lambda_{1}$ in $\vec{v}$ and the changes of $\lambda_{2}$ in $\vec{v}_{\perp}$, which are inevitably competing (two-dimensional effects), the magnitude of $\lambda_{1}$ and $\lambda_{2}$ should be chosen to reflect the relative preference of mesh adaptation along one direction over the other. Generally, smaller $\lambda_{1} / \lambda_{2}$ produces smaller two-dimensional effects and thus better adaptation along just the $\vec{v}$ direction.

It is instructive to consider (3.8) for the monitor functions used in Winslow's method and for the method based upon harmonic maps. For Winslow's method, (3.8) becomes (1.6) with

$$
\lambda_{2}=\lambda_{1}=w(x, y)
$$

In this case, $\vec{v}$ can be chosen as any unit vector. Coordinate lines are compressed or expanded in the $\vec{v}$ direction if $w$ changes rapidly in this direction and otherwise are relatively equally spaced. Thus, the direction in which $w=w(x, y)$ changes most rapidly is the one in which mesh lines are also compressed or expanded most. For the method based upon harmonic maps, we have

$$
\left\{\begin{array}{l}
\lambda_{1}=\sqrt{\alpha_{1} / \alpha_{2}}, \quad \lambda_{2}=\sqrt{\alpha_{2} / \alpha_{1}}, \\
\vec{v}=\text { the normalized eigenvector associated with } \alpha_{1},
\end{array}\right.
$$

where $\alpha_{1}$ and $\alpha_{2}$ are the eigenvalues of the matrix $M$ (see (1.7)). Unlike for Winslow's map, the eigendecomposition (3.1) is unique for the harmonic map unless $\alpha_{1}=\alpha_{2}$. Thus, the mesh adaptation can be expected to occur mainly in the directions of $\vec{v}$ and its orthogonal complement.

One class of mesh adaptation methods uses the arclength-like monitor function $[10,11,19]$. That is, for a physical solution $u=u(x, y), G$ is defined through (1.8) with $M=I+\nabla u \nabla u^{T}$. In this case, the eigenvalues are $\alpha_{1}=1+|\nabla u|^{2}$ and $\alpha_{2}=1$, and the corresponding eigenvectors are $\nabla u /|\nabla u|$ and its orthogonal complement. Motivated by this arclength-like monitor function, we can construct a class of monitor functions with

$$
\left\{\begin{array}{l}
\vec{v}=\nabla u /|\nabla u| \\
\lambda_{1}=\sqrt{1+|\nabla u|^{2}} \\
\lambda_{2}=\text { a function of } \lambda_{1}
\end{array}\right.
$$

to perform mesh adaptation in the gradient direction of $u$. The choices for $\lambda_{2}$ which correspond to Winslow's method and the method based upon harmonic maps are, respectively, $\lambda_{2}=\lambda_{1}$ and $\lambda_{2}=\frac{1}{\lambda_{1}}$. For problems in which $u$ has steep fronts or even discontinuities, $\lambda_{1}$ and $\lambda_{2}$ change much faster in the gradient direction than in the tangential direction. It can be expected that with the choice (3.11), coordinate line compression and expansion will mainly occur in the gradient direction. Furthermore, since the ratio of $\lambda_{1}$ to $\lambda_{2}$ is smaller for Winslow's method than for the one based upon harmonic maps (i.e., $\frac{\lambda_{1}}{\lambda_{2}}=1$ versus $\frac{\lambda_{1}}{\lambda_{2}}=\lambda_{1}^{2}$ ), the two-dimensional effects on the mesh adaptation in $\vec{v}$ will generally be less significant for the former method than 
the latter. In other words, the mesh adaptation in this direction is more like onedimensional adaptation for Winslow's method than for the one based upon harmonic maps. On the other hand, adaptation with the method based upon harmonic maps has a stronger two-dimensional coupling, and our experience has shown that in this case the control of skewness of the mesh can be easier.

It is important to realize that this analysis is a local one. Other factors, such as the shapes of the physical and computational domains and the grid point distributions on boundary, can also strongly influence the mesh adaptation in two dimensions. The influence of the boundary on the elliptic mesh generation is discussed in [19].

To conclude this section, we observe that the above analysis can also be applied to interpret the widely used Poisson mesh generation system

$$
\nabla^{2} \xi=P, \quad \nabla^{2} \eta=Q
$$

where $P$ and $Q$ are control functions, as well as other elliptic mesh generation systems. For (3.12) we easily see that a positive (or negative) value of the Laplacian of one of the curvilinear coordinates implies that the contour lines for that coordinate will shift in the increasing (or decreasing) direction of that coordinate. This result is also obtained in [19] by generalizing their analysis of the mesh behavior near boundaries.

4. Numerical experiments. Numerical results obtained using the mesh equation (1.4) together with Dirichlet boundary conditions (i.e., mesh points fixed along the boundary $\partial \Omega_{p}$ ) are presented in this section. Three examples are chosen to demonstrate the analysis of the previous section. The monitor function is defined using (3.8) with $\vec{v}$ and $\lambda_{1}$ to be chosen and with $\lambda_{2}$ being a function of $\lambda_{1}$. For the first two examples, $\vec{v}$ and $\lambda_{1}$ are artificially designed to illustrate our mesh adaptation analysis. The third is somewhat more realistic, with a "physical solution" prescribed and the complex effects on grid adaptation more in display.

For simplicity, both the physical and computational domains are chosen as the unit square. The mesh equation (1.4) is converted to a so-called moving mesh PDE (MMPDE) [10, 11], a time-dependent PDE with the desired solution of (1.4) as its steady state solution. This MMPDE is discretized in space using central finite differences on a uniform mesh in the computational domain. The resulting system of ordinary differential equations is solved using an ADI-like scheme spatial eigenvalue approximate factorization (SEAF), integrating it until there is little mesh movement, viz., until the $L_{2}$ norm of the difference between two consecutive solutions is less than $10^{-3}$. Also, a low pass filter is applied twice to the monitor function in order to give a smoother mesh and improve the convergence of the scheme. Since our purpose here is to show how the monitor function affects the behavior of the generated mesh and not to study the details of the numerical solution process itself, we refer the interested reader to $[10,11]$ for the details of the finite difference discretization of (1.4) and the SEAF scheme.

The computations were performed on Silicon Graphics workstations, an Indigo2 and an Onyx2 with double precision algorithms.

Example 4.1. For the first example, we choose

$$
\left\{\begin{array}{l}
\vec{v}=\frac{1}{\sqrt{2}}\left[\begin{array}{l}
1 \\
1
\end{array}\right], \quad \vec{v}_{\perp}=\frac{1}{\sqrt{2}}\left[\begin{array}{c}
1 \\
-1
\end{array}\right], \\
\lambda_{1}=1+10 \operatorname{sech}\left(50(x+y-1)^{2}\right) .
\end{array}\right.
$$




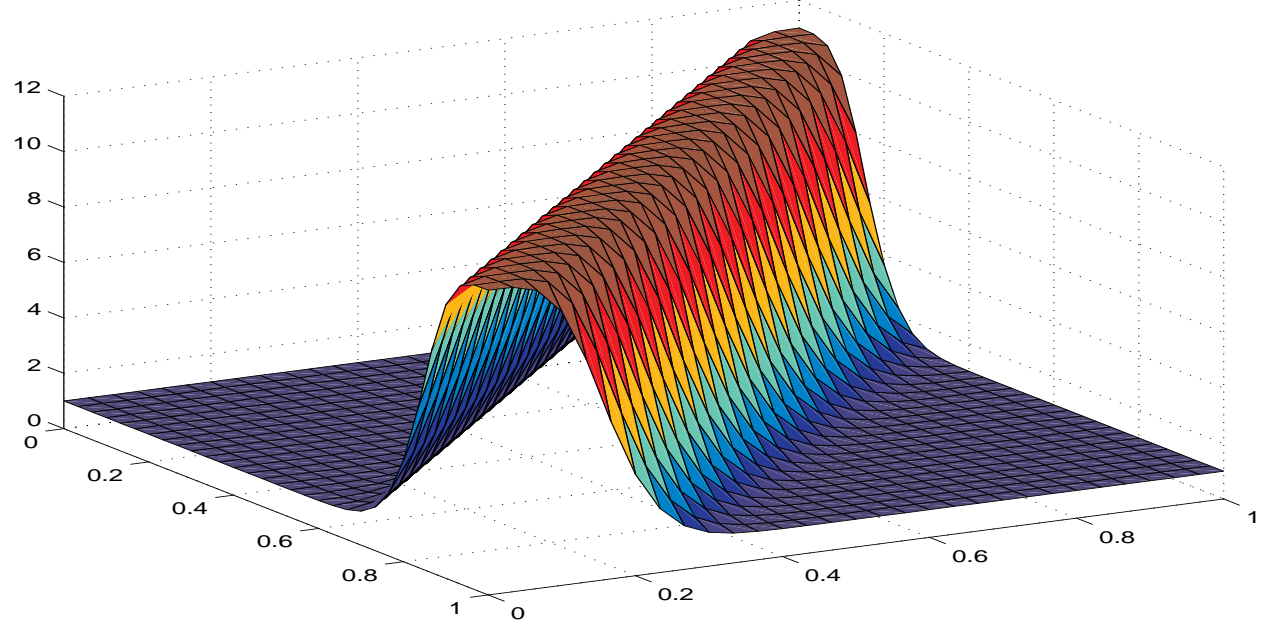

FIG. 4.1. The function $\lambda_{1}$ used in Example 4.1.

Note that the desired direction of mesh adaptation $\vec{v}$ is constant. The function $\lambda_{1}$ changes fastest in this direction and is constant in the perpendicular direction $\vec{v}_{\perp}$. The function $\lambda_{1}$ is shown in Figure 4.1. Along the line $y=x$, for instance, the function $\lambda_{1}$ increases until the center point $(0.5,0.5)$ and then decreases. If mesh adaptation along this line (or direction) is one-dimensional, then from one-dimensional equidistribution arguments (e.g., see [12]) we know that the mesh density increases until this center point and then decreases, so the mesh is densest near the center. On the other hand, from the analysis in the previous section, the change in $\lambda_{1}$ will cause coordinate lines to compress in the $\vec{v}$ direction, and this will compete with the two-dimensional effect resulting from the ellipticity which tends to space coordinate lines equally. Note that in this example the other two-dimensional effects do not take effect because both $v$ and $v_{\perp}$ are constant and $\left(\partial \lambda_{2}\right) /\left(\partial \vec{v}_{\perp}\right)=0$. Moreover, we cannot expect there to be significant mesh adaptation in the $\vec{v}_{\perp}$ direction because, once again, $\left(\partial \lambda_{2}\right) /\left(\partial \vec{v}_{\perp}\right)=0$.

Figure 4.2 shows the $30 \times 30$ meshes generated using four different values of $\lambda_{2}$ : (a) $\lambda_{2}=1 / \lambda_{1}$ (the method based upon harmonic maps), (b) $\lambda_{2}=0.1 \lambda_{1}$, (c) $\lambda_{2}=\lambda_{1}$ (Winslow's method), and (d) $\lambda_{2}=10 \lambda_{1}$. For the four cases, the compression of mesh lines in the $\vec{v}$ direction and basic absence of compression and expansion in the $\vec{v}_{\perp}$ direction can be clearly seen. Recall that the two-dimensional effect on the mesh adaptation in the $\vec{v}$ direction is stronger for the harmonic map than for Winslow's map. For all except the harmonic map case, the mesh is concentrating along the $\vec{v}$ direction as one would expect from one-dimensional mesh adaptation. However, from Figure $4.2 \mathrm{a}$ we see that the mesh adaptation, say along the line $y=x$, is obviously not one dimensional. This is caused by the two-dimensional effect which tends to space mesh lines more equally. This two-dimensional effect can also be seen by comparing Figure $4.2 \mathrm{~b}$ and Figure $4.2 \mathrm{~d}$, corresponding to $\lambda_{1} / \lambda_{2}=10$ and 0.1 , respectively. From our analysis, the two-dimensional effect on the mesh adaptation in the $\vec{v}$ direction is stronger in the former case than in the latter. As a result, the mesh line compression in this direction is weaker in Figure 4.2b than in Figure 4.2d. Finally, it is worth pointing out that for all the cases, mesh lines tend to be uniform near the boundary, particularly near the top-left and bottom-right corners, due to the influence of the uniform boundary point distribution. 

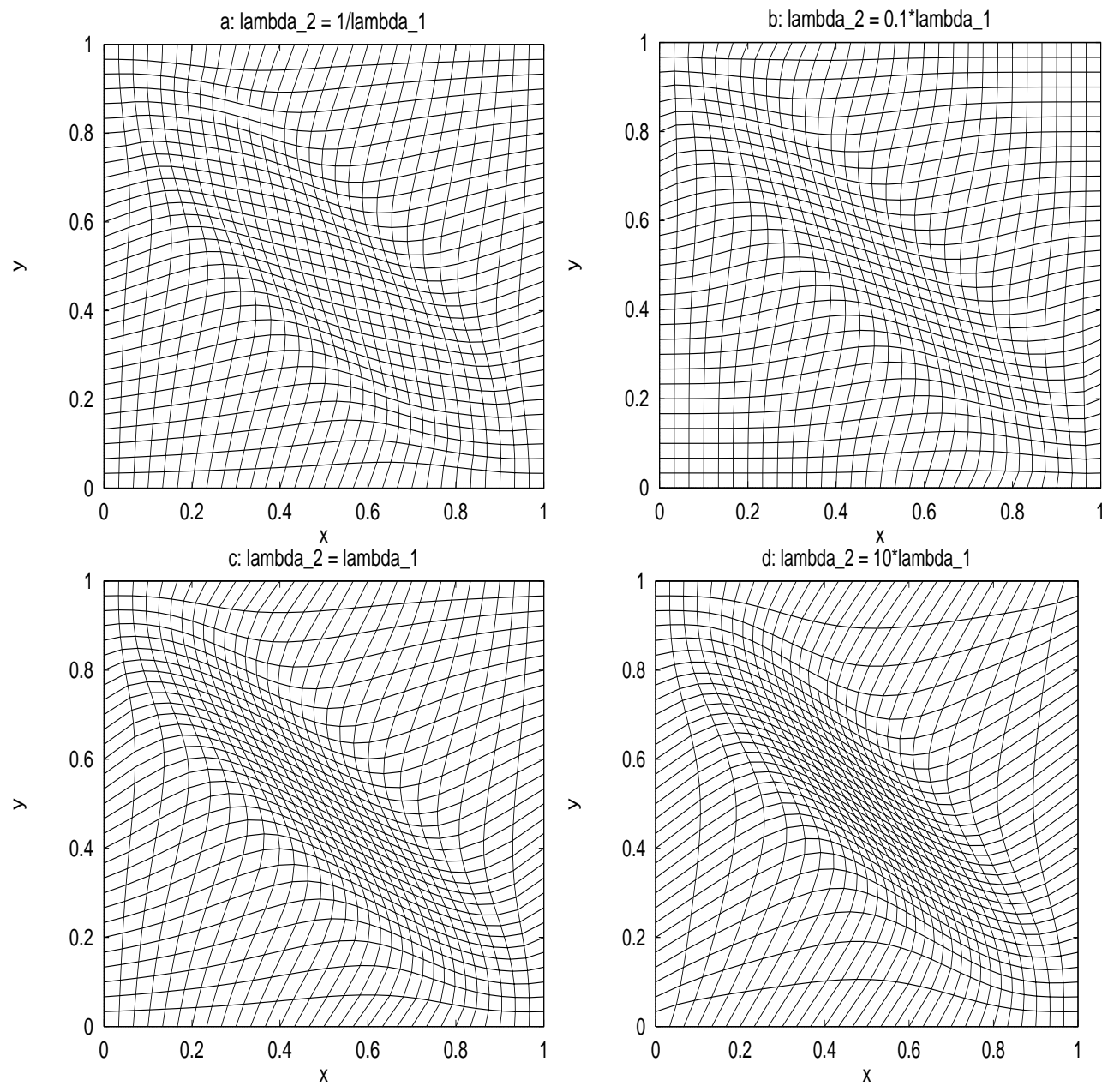

FIG. 4.2. The meshes obtained for Example 4.1 with different $\lambda_{2}$ : (a) $\lambda_{2}=1 / \lambda_{1}$ (method based upon harmonic maps), (b) $\lambda_{2}=0.1 \lambda_{1}$, (c) $\lambda_{2}=\lambda_{1}$ (Winslow's method), (d) $\lambda_{2}=10 \lambda_{1}$.

Example 4.2. Our second example is very similar to the first example except the function $\lambda_{1}$ is now chosen such that its fastest variation direction changes:

$$
\left\{\begin{array}{l}
\vec{v}=\frac{1}{\sqrt{2}}\left[\begin{array}{l}
1 \\
1
\end{array}\right], \quad \vec{v}_{\perp}=\frac{1}{\sqrt{2}}\left[\begin{array}{c}
1 \\
-1
\end{array}\right], \\
\lambda_{1}=1+10 \operatorname{sech}\left(50\left((x-0.5)^{2}+(y-0.5)^{2}-0.3^{2}\right)\right) .
\end{array}\right.
$$

Figure 4.3 shows the meshes generated using four different choices of $\lambda_{2}$. For the harmonic map shown in Figure 4.3a, mesh lines are compressed in the $\vec{v}$ direction in the area around the circle $(x-0.5)^{2}+(y-0.5)^{2}=0.3^{2}$, as one would expect from the analysis in the previous section. In the perpendicular $\vec{v}_{\perp}$ direction, since $\lambda_{2}$ $\left(\equiv 1 / \lambda_{1}\right)$ first decreases and then increases around the circle, mesh lines are expanded. From the analysis we also know that the two-dimensional effect is stronger in the $\vec{v}$ direction than the $\vec{v}_{\perp}$ direction, although it is hard to detect from this figure precisely what the effect is. For Winslow's map, there is no preferred direction of adaptation. Since $\lambda_{1}$ changes in every direction, mesh lines are compressed uniformly around the 



FIG. 4.3. The meshes obtained for Example 4.2 with different $\lambda_{2}$ : (a) $\lambda_{2}=1 / \lambda_{1}$ (method based upon harmonic maps), (b) $\lambda_{2}=0.1 \lambda_{1}$, (c) $\lambda_{2}=\lambda_{1}$ (Winslow's method), (d) $\lambda_{2}=10 \lambda_{1}$.

circle (see Figure 4.3c). For the other two cases, $\lambda_{2}$ is chosen to be $0.1 \lambda_{1}$ and $10 \lambda_{1}$, respectively. As predicted, mesh lines are compressed in both the $\vec{v}$ and $\vec{v}_{\perp}$ direction. However, since the ratio $\lambda_{1} / \lambda_{2}$ is 10 in the first case, the two-dimensional effect is stronger in the $\vec{v}$ direction than the $\vec{v}_{\perp}$ direction. Thus, the mesh line compression is less in the $\vec{v}$ direction than the $\vec{v}_{\perp}$ direction (see Figure $4.3 \mathrm{~b}$ ). Conversely, for the case in Figure 4.3d, the ratio $\lambda_{1} / \lambda_{2}$ is 0.1 , and, therefore, the mesh line compression is stronger in the $\vec{v}$ direction than the $\vec{v}_{\perp}$ direction.

Example 4.3. The third example is more realistic than the previous two. Here, we assume that the "physical" solution $u$ is given and the desired mesh adaptation direction is taken as the gradient direction of the solution. According to (3.11), we define $G$ with

$$
\left\{\begin{array}{l}
u(x, y)=\operatorname{sech}\left(50\left((x-0.5)^{2}+(y-0.5)^{2}-0.3^{2}\right)\right) \\
\vec{v}=\nabla u /|\nabla u| \\
\lambda_{1}=\sqrt{1+|\nabla u|^{2}}
\end{array}\right.
$$



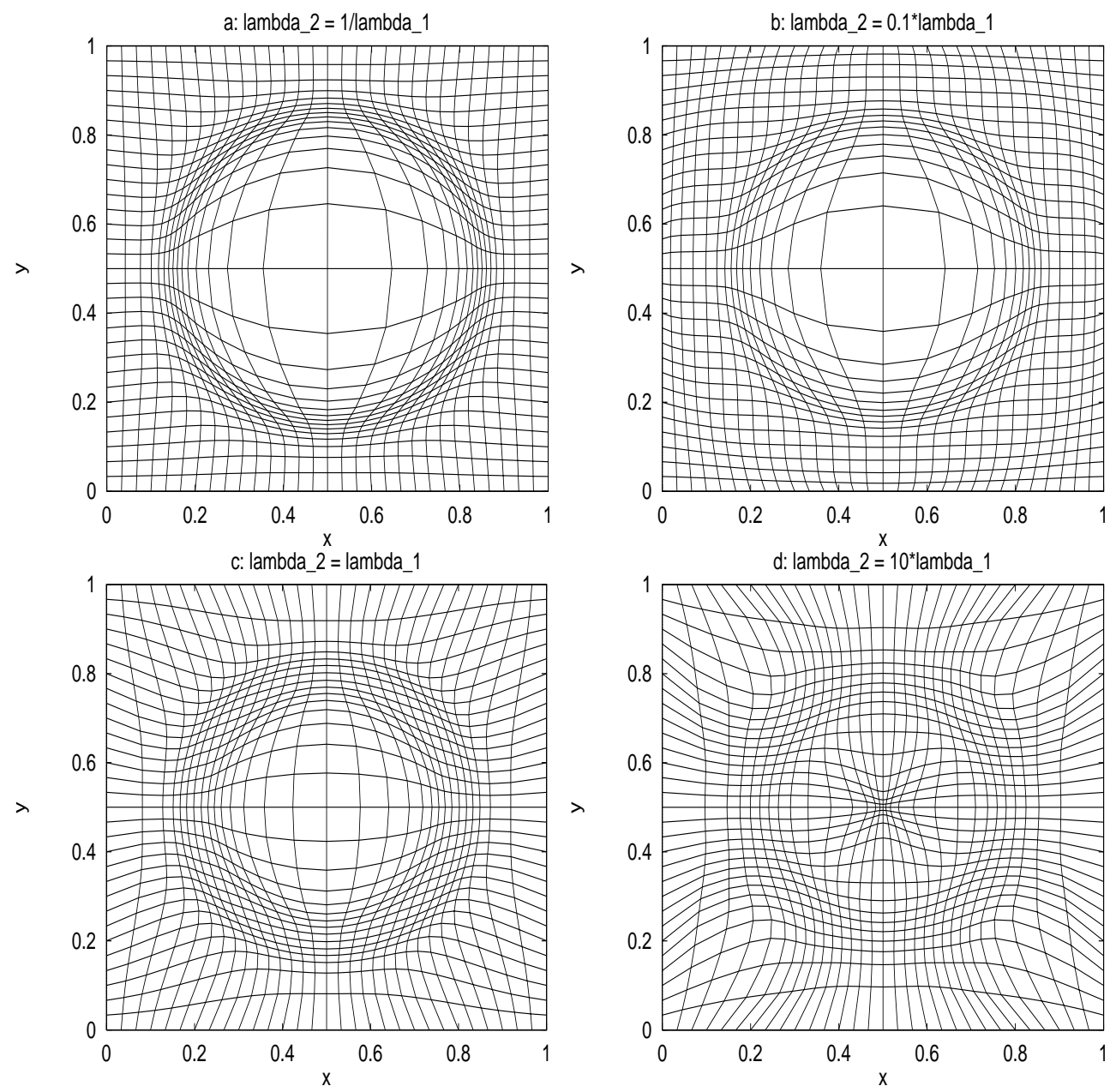

FIG. 4.4. The meshes obtained for Example 4.3 with different $\lambda_{2}$ : (a) $\lambda_{2}=1 / \lambda_{1}$ (method based upon harmonic maps), (b) $\lambda_{2}=0.1 \lambda_{1}$, (c) $\lambda_{2}=\lambda_{1}$ (Winslow's method), (d) $\lambda_{2}=10 \lambda_{1}$.

Note that the function $\lambda_{1}$ behaves similarly to that in Example 4.2, changing most significantly across the circle $(x-0.5)^{2}+(y-0.5)^{2}=0.3^{2}$. The meshes obtained with four different choices of $\lambda_{2}$ are shown in Figure 4.4. As expected from the theoretical analysis, mesh line compression occurs mainly in the radial direction oriented from the center $(0.5,0.5)$ in all the cases. It is easy to show that $\left(\partial \lambda_{2}\right) /\left(\partial \vec{v}_{\perp}\right)=0$ since $\lambda_{2}$ is a function of $\lambda_{1}$. Thus the two-dimensional effects only come from the ellipticity and the change of $\vec{v}$. However, in this case they are much more complicated than in the previous examples. They will be analyzed in the next section.

5. Mesh adaptation on the unit disk. In the previous two sections we have investigated theoretically and numerically how the qualitative behavior of the mesh is related to the change in the eigensystems of the monitor function. The twodimensional effect is extremely complicated to analyze for the general case. To get insight into this effect, we consider the special case where $\Omega_{p}$ and $\Omega_{c}$ are unit disks, and the monitor function is given by (3.8) with 


$$
\left\{\begin{array}{l}
\vec{v}=(\cos \theta, \sin \theta)^{T}, \quad \vec{v}_{\perp}=(-\sin \theta, \cos \theta)^{T}, \\
\lambda_{1}=\lambda_{1}(r), \quad \lambda_{2}=\lambda_{2}(r) \quad \text { both positive and bounded above. }
\end{array}\right.
$$

Here $(r, \theta)$ are polar coordinates in $\Omega_{p}, \vec{v}$ is the unit radial vector, and $\vec{v}_{\perp}$ is the unit angular vector. Under these assumptions, the mesh adaptation problem has angular symmetry, so the mesh equations can be reduced to ordinary differential equations.

In fact, using the identities

$$
\left\{\begin{array}{l}
\nabla=\vec{v} \frac{\partial}{\partial r}+\frac{1}{r} \vec{v}_{\perp} \frac{\partial}{\partial \theta}, \\
\frac{\partial}{\partial \vec{v}}=\frac{\partial}{\partial r}, \quad \frac{\partial}{\partial \vec{v}_{\perp}}=\frac{1}{r} \frac{\partial}{\partial \theta}
\end{array}\right.
$$

the mesh equations (3.5) and (3.6) become

$$
\begin{aligned}
& \frac{1}{\lambda_{1}} \frac{\partial^{2} \xi}{\partial r^{2}}+\frac{1}{\lambda_{2} r^{2}} \frac{\partial^{2} \xi}{\partial \theta^{2}}=\frac{1}{\lambda_{1}}\left(\frac{1}{\lambda_{1}} \frac{d \lambda_{1}}{d r}-\frac{1}{r}\right) \frac{\partial \xi}{\partial r}+\frac{1}{\lambda_{2} r^{2}}(0-0) \frac{\partial \xi}{\partial \theta}, \\
& \frac{1}{\lambda_{1}} \frac{\partial^{2} \eta}{\partial r^{2}}+\frac{1}{\lambda_{2} r^{2}} \frac{\partial^{2} \eta}{\partial \theta^{2}}=\frac{1}{\lambda_{1}}\left(\frac{1}{\lambda_{1}} \frac{d \lambda_{1}}{d r}-\frac{1}{r}\right) \frac{\partial \eta}{\partial r}+\frac{1}{\lambda_{2} r^{2}}(0-0) \frac{\partial \eta}{\partial \theta} .
\end{aligned}
$$

The two-dimensional effects on the mesh adaptation in the radial direction result only from the change in $\vec{v}((\nabla \cdot \vec{v})=1 / r)$ and the ellipticity (from the second term of each of the equations in (5.3)). Using the polar coordinates $(R, \Theta)$ in $\Omega_{c}$, so

$$
\xi=R \cos \Theta, \quad \eta=R \sin \Theta,
$$

we have by symmetry that $\Theta=\theta$ and $R=R(r)$. Then (5.3) leads to

$$
\frac{d^{2} R}{d r^{2}}=\left(\frac{1}{\lambda_{1}} \frac{d \lambda_{1}}{d r}\right) \frac{d R}{d r}-\left(\frac{1}{r}\right) \frac{d R}{d r}+\left(\frac{\lambda_{1}}{\lambda_{2}}\right) \frac{R}{r^{2}} .
$$

It is easy to show from this equation and the boundary conditions $R(0)=0$ and $R(1)=1$ that

$$
R=R(r) \in(0,1), \quad \frac{d R}{d r}(r)>0 \text { for } r \in(0,1) .
$$

The mesh point density function in the radial direction is $\frac{d R}{d r}$, and the first, second, and third terms on the right-hand side of (5.5) represent the effects of the change in $\lambda_{1}$, the change in $\vec{v}$, and the ellipticity of the underlying system. We can readily conclude that as $r$ increases, the two-dimensional effect from a change of $\vec{v}$ corresponds to a decrease in the density function and the ellipticity term causes an increase in the density function. In particular, mesh point density near the origin will be increased by change in $\vec{v}$ and decreased by the ellipticity. Of course, these two-dimensional effects compete with one another and with the effect caused by the relative change in $\lambda_{1}$. The degree of the effect from ellipticity is controlled by the ratio $\lambda_{1} / \lambda_{2}$. To see this, we consider the case where $\lambda_{1}$ and $\lambda_{2}$ are constants, for which an analytical solution of (5.5) is available, viz. $R(r)=r \sqrt{\lambda_{1} / \lambda_{2}}$. Note that for $\frac{\lambda_{1}}{\lambda_{2}}=4,1$, and $\frac{1}{4}$, $\frac{d R}{d r}=2 r, 1$, and $\frac{1}{2} r^{-1 / 2}$, respectively. The smaller the ratio $\frac{\lambda_{1}}{\lambda_{2}}$, the stronger the effect of the change in $\vec{v}$, and therefore the denser the mesh will be near the origin.

For Example 4.3, the physical domain is not a disk, but because the boundary point distribution does not strongly affect the adaptation inside the domain and 
because $\lambda_{1}$ and $\vec{v}$ behave similarly to those in this section, one can expect that the above qualitative arguments should remain valid. For the harmonic map shown in Figure 4.4a, since $\lambda_{1}$ changes significantly in $r$ ( $r$ is the distance to the point $(0.5,0.5)$ ) and the ratio $\lambda_{1} / \lambda_{2}$ is large (i.e., $\left.\lambda_{1}^{2}\right)$ near $r=0.3$, the adaptation and the two-dimensional effect from ellipticity dominate the mesh line placement. Thus, we can see that the mesh points are pulled away from point $(0.5,0.5)$ (the ellipticity effect) and are denser near $r=0.3$ (the adaptation effect). On the other hand, for the case shown in Figure $4.4 \mathrm{~d}$, the ratio $\lambda_{1} / \lambda_{2}$ is 0.1 (small), and the adaptation and the two-dimensional effect from the change in $\vec{v}$ dominate the mesh line placement. Thus, the mesh points are concentrated near the center point $(0.5,0.5)$ (the effect of the change in $\vec{v}$ ) and in the area where $r \approx 0.3$ (the effect of adaptation). The case shown in Figure 4.4b is similar to that in Figure 4.4a. For the case shown in Figure 4.4c, the two-dimensional effects from the change in $\vec{v}$ and the ellipticity appear to be more or less balanced.

6. The three-dimensional case. We briefly discuss the three-dimensional case. Denote the physical and computational coordinates by $\vec{x}=\left(x^{1}, x^{2}, x^{3}\right)^{T}$ and $\vec{\xi}=$ $\left(\xi^{1}, \xi^{2}, \xi^{3}\right)^{T}$, respectively. The general functional corresponding to (1.2) is

$$
I[\vec{\xi}]=\frac{1}{2} \int_{\Omega_{p}} d \vec{x} \sum_{i}\left(\nabla \xi^{i}\right)^{T} G^{-1} \nabla \xi^{i}
$$

where $G^{-1}$ is a symmetric positive definite matrix (monitor function). The EulerLagrange equation is

$$
\nabla \cdot\left(G^{-1} \nabla \xi^{i}\right)=0
$$

If $\left(\lambda_{i}, \vec{v}_{i}\right), i=1,2,3$ are the three pairs of eigenvalues and eigenvectors of $G$, it has the eigendecomposition

$$
G=\sum_{i} \lambda_{i} \vec{v}_{i} \vec{v}_{i}^{T}
$$

The general functional (6.1) can then be rewritten as

$$
I[\vec{\xi}]=\frac{1}{2} \int_{\Omega_{p}} d \vec{x} \sum_{i, j} \frac{1}{\lambda_{i}}\left|\frac{\partial \xi^{j}}{\partial \vec{v}_{i}}\right|^{2},
$$

and the Euler-Lagrange equation becomes

$$
\sum_{j} \frac{1}{\lambda_{j}} \frac{\partial^{2} \xi^{i}}{\partial \vec{v}_{j}^{2}}=\sum_{j} \frac{1}{\lambda_{j}}\left(\frac{1}{\lambda_{j}} \frac{\partial \lambda_{j}}{\partial \vec{v}_{j}}-\nabla \cdot \vec{v}_{j}\right) \frac{\partial \xi^{i}}{\partial \vec{v}_{j}} .
$$

An extension of the analysis in sections 2 and 3 enables us to similarly conclude that (i) coordinate lines can be expected to compress and/or expand in the $\vec{v}_{i}$ direction if the function $\lambda_{i}$ changes significantly in this direction; (ii) the compression and/or expansion (i.e., adaptation) of coordinate lines in the $\vec{v}_{i}$ direction have to compete with the three-dimensional effects resulting from the changes in $\vec{v}_{1}, \vec{v}_{2}$, and $\vec{v}_{3}$, the relative change in $\lambda_{j}$ along the direction of $\vec{v}_{j}(j=1,2,3$ and $j \neq i)$, and the ellipticity of the underlying system; (iii) among the three-dimensional effects, ellipticity tends to give more even placement of mesh lines. All of the three-dimensional effects on the mesh adaptation in $\vec{v}_{i}$ except that from the change in $\vec{v}_{i}$ can be controlled by 
controlling the ratios $\lambda_{i} / \lambda_{j}(j=1,2,3$ and $j \neq i)$. More specifically, the smaller the ratios $\lambda_{i} / \lambda_{j}(j=1,2,3$ and $j \neq i)$, the weaker these three-dimensional effects and the more dominant the effects of changes in $\lambda_{i}$ and $\vec{v}_{i}$ are on the adaptation along $\vec{v}_{i}$ direction.

Motivated by this analysis, it is natural to define the monitor function using (6.3) with

$$
\left\{\begin{array}{l}
\vec{v}_{1}=\nabla u /|\nabla u|, \\
\vec{v}_{2}, \vec{v}_{3} \text { are orthogonal complements of } \vec{v}_{1}, \\
\lambda_{1}=\sqrt{1+|\nabla u|^{2}}, \\
\lambda_{2} \text { and } \lambda_{3} \text { are functions of } \lambda_{1},
\end{array}\right.
$$

where $u=u\left(x_{1}, x_{2}, x_{3}\right)$ is the solution of the physical PDE. One of course has considerable flexibility in these choices. For instance, curvature information for $u$ could also be included in the definition of $\lambda_{1}$. What is important is that the eigenvectors and eigenvalues of the monitor function determine, respectively, the directions of adaptation and strength of compression/expansion in these directions, and this is used to tailor $G$ to meet the needs of a particular application area.

7. Conclusions. In the previous sections, we have studied the effect of the monitor function on the behavior of the mesh generated with a variational approach. The study has been carried out using Green's function for elliptic problems. Numerical results have also been presented for illustrative purposes.

The main conclusion of this study is that a significant change (first increasing and then decreasing, or vice versa) in an eigenvalue $\lambda_{1}$ of the monitor function $G$ along the corresponding eigendirection $\vec{v}_{1}$ will result in adaptation of coordinate lines along this direction. Meanwhile, this adaptation will compete with far more complicated two-dimensional effects, including the effect of the ellipticity and those from changes in eigenvectors and another eigenvalue $\lambda_{2}$ of $G$. Fortunately, most of these twodimensional effects can be controlled by the ratio $\lambda_{1} / \lambda_{2}$. That is, the smaller this ratio, the weaker the two-dimensional effects and the more dominant the effect that change in $\lambda_{1}$ has on the adaptation along the $\vec{v}_{1}$ direction. The same analysis holds along the $\vec{v}_{2}$ direction.

Based upon this analysis, general guidelines for defining monitor functions to achieve the desired mesh adaptation in practical computation have been given. In fact, a monitor function can often be naturally defined in terms of suitably chosen $\lambda_{1}$, $\lambda_{2}, \vec{v}_{1}$, and $\vec{v}_{2}$ through formula (3.1) or (3.8). Specifically, when one wishes mesh concentration or expansion to occur along a given direction, e.g., the streamline direction in a convection-diffusion problem or the gradient direction for a general problem, then one may choose this direction as the eigenvector $\vec{v}$ and choose $\lambda_{1}$ increasing and then decreasing (or vice versa) in certain regions to give mesh concentration (or expansion) along the $\vec{v}$ direction. There are a variety of natural ways in which $\lambda_{2}$ can be chosen as a function of $\lambda_{1}$. Last, the class of monitor functions given in (3.11) or (6.6) is useful when one wants the coordinate lines to adapt along the gradient direction of a physical variable $u$.

We would like to point out that the results obtained in this study are only qualitative. Besides the competing effects of the mesh adaptation along the two eigendirections, mesh adaptation is also greatly affected by the shapes of the physical and computational domains and the grid point distribution on the boundaries. These factors make the mesh adaptation procedure very complicated. Many difficulties still 
remain in predicting the precise behavior of adaptive meshes. Nevertheless, it is our hope that this study will provide a better understanding of monitor functions thereby providing some guidelines for one to choose them more easily and will be a useful complement to related recent work in grid generation such as that in $[3,13]$.

\section{REFERENCES}

[1] D. A. Anderson, Equidistribution schemes, Poisson generators, and adaptive grids, Appl. Math. Comput., 24 (1987), pp. 211-227.

[2] D. A. Anderson, Grid cell volume control with an adaptive grid generator, Appl. Math. Comput., 35 (1990), pp. 209-217.

[3] J. U. Brackbill, An adaptive grid with direction control, J. Comput. Phys., 108 (1993), pp. 38-50.

[4] J. U. Brackbill and J. S. Saltzman, Adaptive zoning for singular problems in two dimensions, J. Comput. Phys., 46 (1982), pp. 342-368.

[5] R. Courant and D. Hilbert, Methods of Mathematical Physics, Vol. II, John Wiley and Sons, New York, 1962.

[6] A.S. Dvinsky, Adaptive grid generation from harmonic maps on Riemannian manifolds, J. Comput. Phys., 95 (1991), pp. 450-476.

[7] P. R. Eiseman, Adaptive grid generation, Comput. Methods Appl. Mech. Engrg., 64 (1987), pp. 321-376.

[8] R. HAGMEIJER, Grid adaption based on modified anisotropic diffusion equations formulated in the parametric domain, J. Comput. Phys., 115 (1994), pp. 169-183.

[9] R. Hamilton, Harmonic Maps of Manifolds with Boundary, Lecture Notes in Mathematics 471, Springer-Verlag, Berlin, New York, 1975.

[10] W. Huang and R. D. Russell, A high dimensional moving mesh strategy, Appl. Numer. Math., 26 (1998), pp. 63-76.

[11] W. Huang And R. D. Russell, Moving mesh strategy based on a gradient flow equation for two-dimensional problems, SIAM J. Sci. Comput., to appear.

[12] W. Huang, Y. Ren, And R. D. Russell, Moving mesh partial differential equations (MMPDEs) based upon the equidistribution principle, SIAM J. Numer Anal., 31 (1994), pp. 709-730.

[13] P. Knupp, Mesh generation using vector-fields, J. Comput. Phys., 119 (1995), pp. 142-148.

[14] P. Knupp And S. Steinberg, Fundamentals of Grid Generation, CRC Press, Boca Raton, FL, 1994.

[15] G. Liao, A study of regularity problem of harmonic maps, Pacific J. Math., 131 (1988), pp. 291-302.

[16] G. Liao And N. Smale, Harmonic maps with nontrivial higher-dimensional singularities, Lecture Notes in Pure and Appl. Math. 144, Dekker, New York, (1993), pp. 79-89.

[17] M. H. Protter and H. F. Weinberger, Maximum Principles in Differential Equations, Prentice-Hall, Inc., Englewood Cliffs, NJ, 1967.

[18] Y. Tu And J. F. Thompson, Three-dimensional solution-adaptive grid generation on composite configurations, AIAA J., 29 (1991), pp. 2025-2026.

[19] J. F. Thompson, Z. U. A. Warsi, And C. W. Mastin, Numerical Grid Generation, Foundation and Applications, North-Holland, New York, 1985.

[20] Z. U. A. Warsi And J. F. Thompson, Application of variational methods in the fixed and adaptive grid generation, Comput. Math. Appl., 19 (1990), pp. 31-41.

[21] A. Winslow, Numerical solution of the quasi-linear Poisson equation in a nonuniform triangle mesh, J. Comput. Phys., 1 (1967), pp. 149-172. 